\title{
Temperature Dependence of the Optical Activity of Pure and Deuterated Rochelle Salt Crystals
}

\author{
M. Koralewski And M. Szafrański \\ Institute of Physics, A. Mickiewicz University \\ Umultowska 85, 61-614 Poznań, Poland
}

(Received March 7, 2002)

\begin{abstract}
Temperature dependence of the optical rotatory power $(\rho)$ along the optical axis of pure and deuterated Rochelle salt single crystals has been given. Three phases of both crystals were examined and found to be optically active. The relation between spontaneous electrogyration $\left(\rho_{s}\right)$ and spontaneous polarization $\left(P_{\mathrm{s}}\right)$ can be described by $\rho_{\mathrm{s}} \propto P_{\mathrm{s}}^{2}$. Moreover, the antipolar domains of pure and denterated ferroelectric Rochelle salt crystals were found of the rotation twin-type not occurring in the enantiomorphic form, in contrast to those in other ferroelectric crystals, e.g., TGS-type and $\mathrm{Pb}_{5} \mathrm{Ge}_{3} \mathrm{O}_{11}$.
\end{abstract}

PACS numbers: $77.80 . \mathrm{Bh}, 78.20 . \mathrm{Ek}$

\section{Introduction}

Sodium potassium tartrate tetrahydrate $\left(\mathrm{NaKC}_{4} \mathrm{H}_{4} \mathrm{O}_{6} \cdot 4 \mathrm{H}_{2} \mathrm{O}\right)$, known as Rochelle salt (RS), has been the first ferroelectric material discovered [1]. The crystal structure of RS in two paraelectric phases, below $T_{c_{1}}=255 \mathrm{~K}$ and above $T_{\mathrm{C}_{2}}=297 \mathrm{~K}$, is orthorhombic (space group $P 2_{1} 2_{1} 2$ ); in the intermediate ferroelectric phase it is monoclinic (space group $P 2_{1}$ ) [2]. Deuterated Rochelle salt $\left(\mathrm{NaKC}_{4} \mathrm{H}_{2} \mathrm{D}_{2} \mathrm{O}_{6} \cdot 4 \mathrm{D}_{2} \mathrm{O}\right.$ - DRS) is isostructural to RS but $T_{\mathrm{C}_{1}}=251 \mathrm{~K}$ and $T_{\mathrm{C}_{2}}=308 \mathrm{~K}[2]$.

Optical activity $(\mathrm{OA})$ can occur in all three phases of RS and DRS crystals in agreement with their symmetry. The OA of RS crystal was found by Dufet [3] long before its ferroelectric properties were discovered. Optical rotatory dispersion (ORD) of RS was studied by Kozik [4] at room temperature. Further discussion of 
the ORD and circular dichroism (CD) of the RS as well as DRS crystals at room temperature was reported by Koralewski and Mróz [5].

The first measurements of temperature dependence of OA along the optical axis of RS were performed by Levy [6] who related OA directly to the spontaneous polarization $\left(P_{\mathrm{s}}\right)$. However, these results were not confirmed by the later studies of Hermelbracht and Unruh [7], and Vlokh et al. [8]. Vlokh et al. [8] reported that for the electric field $(E)$ greater than the coercive field $\left(E_{\mathrm{C}}\right)$ of RS the optical rotatory power $(\rho)$ does not depend on the sign and the value of the external electric field. The same authors [8] proved that the shape of $\rho(E)$ dependence was close to the hysteresis loop of the butterfly-type, in contrast to the analogous dependence observed for TGS-type [9] and $\mathrm{Pb}_{5} \mathrm{Ge}_{3} \mathrm{O}_{11}$ crystals [10]. A more detailed discussion of the $\rho(E)$ dependence for RS and TGS crystals was given by Kobayashi et al. [11]. These authors established experimentally that the antipolar domains in RS are not enantiomorphic and are united by hemitrope twin mechanism. The most recent measurements of Kobayashi et al. [12] showed that spontaneous gyration in the direction of the ferroelectric $x$-axis was proportional to $P_{\mathrm{s}}^{2}$. For the optical axis direction a similar relation may be expected, however, till now there is no experimental evidence.

The temperature dependence of DRS single crystal as well as the effect of deuteration on $\mathrm{OA}$ have not been discussed up to date, so these and the ambiguities in the hitherto reported temperature measurements and their interpretation have lead us to undertake a study of the temperature dependence of $\mathrm{OA}$ along the optical axis of RS single crystal as well as its deuterated analogue.

\section{Experimental}

Single crystals of RS were grown by slow evaporation of saturated aqueous solution at room temperature. Single crystals of DRS were grown according to the procedure described by Holden and Mason [13]. Both crystals of size of $\sim 20 \times 30 \times 50 \mathrm{~mm}^{3}$ were colourless, transparent, and showed a good optical quality. The degree of deuteration $(D)$ was estimated according to Holden and Mason [13] on the basis of the higher $T_{\mathrm{c}_{2}}$ and lower $T_{\mathrm{C}_{1}}$ Curie points, and found to be $D \approx 90 \%$ and $D \approx 55 \%$ for two different crystals. Crystals with higher $\left(T_{\mathrm{c}_{1}}=252 \mathrm{~K}\right.$ and $\left.T_{\mathrm{C}_{2}}=306.8 \mathrm{~K}\right)$ and lower $\left(T_{\mathrm{C}_{1}}=253.7 \mathrm{~K}\right.$ and $\left.T_{\mathrm{C}_{2}}=300.7 \mathrm{~K}\right)$ degree of deuteration will be labelled as $\mathrm{DRS}_{\mathrm{I}}$ and $\mathrm{DRS}_{\mathrm{II}}$, respectively. The samples were prepared in the form of rods of 5-9 $\mathrm{mm}$ in diameter and $3-7 \mathrm{~mm}$ in length, with the axis parallel to one of the optical axes of the crystal. The direction of the optical axis was determined and controlled during the temperature measurements by means of the conoscopic method. The experimental set-up was the same as described previously $[9,14,15]$. The measurements were carried out using a He-Ne laser $(\lambda=632.8 \mathrm{~nm})$ as a light source. 


\section{Results and discussion}

Measurements of the optical rotatory power of RS and DRS crystals in the ferroelectric phase have been made in the external electric field whose value was increased up to $200 \mathrm{kV} / \mathrm{m}$, thus, much above the coercive field of these crystals $\left(E_{\mathrm{c}}^{\mathrm{RS}} \simeq 20 \mathrm{kV} / \mathrm{m}, E_{\mathrm{c}}^{\mathrm{DRS}} \simeq 40 \mathrm{kV} / \mathrm{m}[2]\right)$, and whose sign was alternated. It was shown that the sign of $\rho$ does not change and only the value of $\rho$ changes slightly when the polarization is reversed. The changes could be described by a butterfly-type hysteresis loop. A typical relation $\rho(E)$ for the crystal DRS II is shown in Fig. 1. The hysteresis loop observed for DRS is similar to the gyration

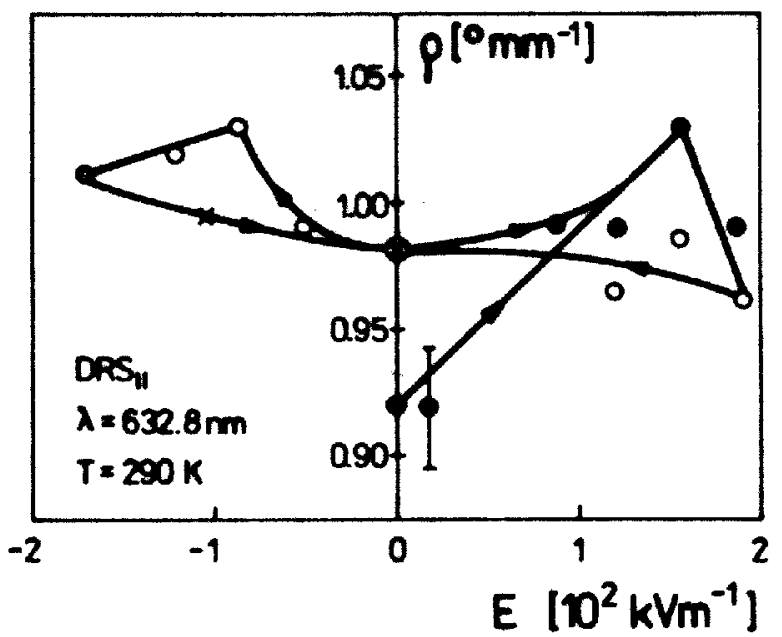

Fig. 1. The hysteresis loop of the optical rotatory power of $\mathrm{DRS}_{\text {II }}$ crystal at $290 \mathrm{~K}$. The closed, open, and crossed points denote the direction (see also arrows) of the electric field changes.

loops reported by Vlokh et al. [8] and Kobayashi et al. [11] for pure RS crystals. Therefore, we can conclude that the domains in DRS are not enantiomorphic similarly as in the RS crystals. This would mean that, according to the nomenclature of Shuvalov et al. [16, 17], the antipolar domains in RS and DRS crystals are of the rotatory twin-type. The asymmetric shape of the hysteresis loop observed by us and by other authors $[8,11]$ indicates a certain instability of the domain structure of RS and DRS crystals, which would at least partly explain a significant scatter of measuring points in the temperature and electric field dependences of $\rho$.

The optical rotatory power of RS and DRS crystals was measured in the temperature range of $245-315 \mathrm{~K}$. For a few temperatures $\rho$ was measured both at $E>E_{c}$ and at $E=0$. The values obtained in these two series of measurements were comparable within the experimental error and were characterized by a similar scatter. Therefore, systematic measurements of $\rho$ in the above temperature 


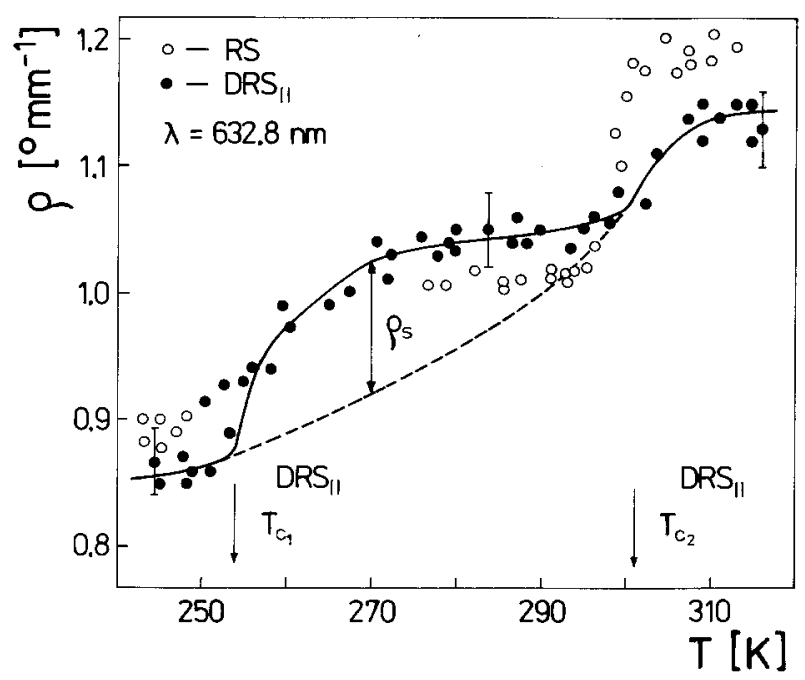

Fig. 2. The optical rotatory power $\rho$ versus temperature for RS and DRS II crystals, $\rho_{\mathrm{S}}$ is the spontaneous optical rotatory power.

range were carried out for $E=0$ after a preliminary multiple polarization reversal of a given sample in the field $E>E_{\mathrm{c}}$. The results are shown in Fig. 2. The observed changes of $\mathrm{OA}$ at the phase transition temperature $T_{\mathrm{c}_{2}}$ are continuous (Fig. 2), which confirms the conclusions following from other data $[2,18]$ that the phase transition is continuous in both studied crystals. In spite of the fact that the temperature changes were very slow and at each temperature the sample was stabilized for about 2 hours before making a series of measurements, the results show a certain scatter of $\rho$ values. This scatter and a significant uncertainty of measurements much exceeding the uncertainty of the measuring set-up is a consequence of the above-mentioned instability of the domain structure, high deliquescence of the crystals, and the process of their decomposition above a certain temperature. The rate of dehydration, that is the loss of crystalline water [19, 20], depends on temperature and pressure conditions. At $309 \mathrm{~K}$ the Rochelle salt decomposes into $\mathrm{K}_{2} \mathrm{C}_{4} \mathrm{H}_{4} \mathrm{O}_{6} \cdot \frac{1}{2} \mathrm{H}_{2} \mathrm{O}$ and $\mathrm{Na}_{2} \mathrm{C}_{4} \mathrm{H}_{4} \mathrm{O}_{6} \cdot 2 \mathrm{H}_{2} \mathrm{O}$, while at $323.5 \mathrm{~K}$ these salts undergo the irreversible decay [19]. This process determines the upper limit of temperatures in the high-temperature paraelectric phase, in which the OA of RS and DRS crystals can be studied. The low-temperature limit was not so drastic, but below $273 \mathrm{~K}$ when the sample was under reduced pressure, in vacuum, the process of dehydration was faster. The occurrence of these processes was limited by all possible means, but a total elimination of their influence on the results of measurements of $\rho$ was impossible.

The uncertainty of measurements and the scatter of the values of $\rho$ for RS and DRS crystals are similar to those obtained by Hermelbracht and Unruh [7], and Vlokh et al. [8] for RS. Our values of $\rho$ determined for RS are in very good 
agreement with the results reported in $[7,8]$ for all the three phases but are contradictory to the results obtained by Levy [6] for the ferroelectric phase. The change $\Delta \rho$ in the range from $+18^{\circ} \mathrm{C}$ to $-18^{\circ} \mathrm{C}$ observed by Levy gave a symmetric bell-shaped curve with a maximum of $-1.6^{\circ} \mathrm{mm}^{-1}$ at $0^{\circ} \mathrm{C}$. The temperature dependence of $\Delta \rho$ Levy interpreted as related to the temperature changes of the spontaneous polarization. As we will show, his supposition about the correlation between $\Delta \rho$ and $P_{\mathrm{s}}$ is correct, but the absolute value of the $\Delta \rho$ changes and their sign are doubtful in a view of our results and those given in $[7,8]$.

The temperature changes of the optical rotatory power of DRS are similar to those observed for RS. In the paraelectric phase the optical rotatory power of DRS is smaller than that of RS while in the ferroelectric phase the reverse relation $\rho^{\mathrm{DRS}}>\rho^{\mathrm{RS}}$ is true (Fig. 2). The influence of deuteration on the optical rotatory power is illustrated in Fig. 3. The reverse relation in the ferroelectric phase can be

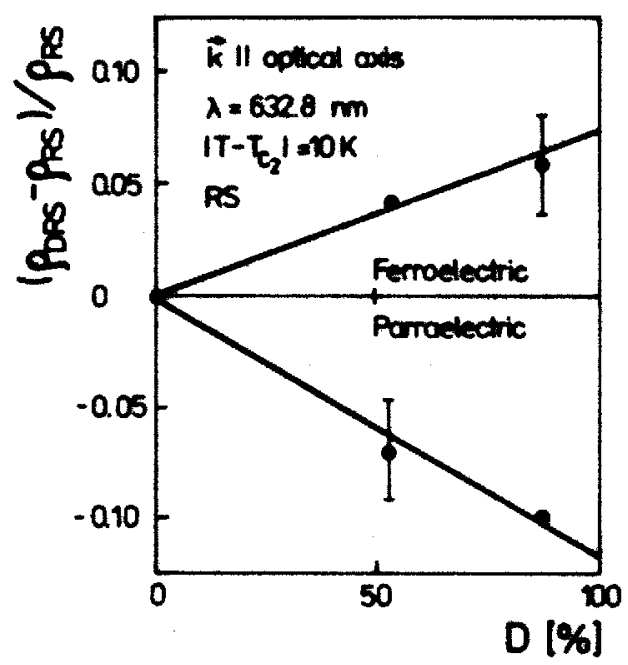

Fig. 3. Changes of the optical rotatory power of the RS crystals in the paraelectric phase at $\left(T-T_{\mathrm{C}_{2}}\right)=10 \mathrm{~K}$ and in the ferroelectric phase at $\left(T_{\mathrm{C}_{2}}-T\right)=10 \mathrm{~K}$ for different degrees of deuteration $D$.

a result of an increase (about 1.5 times) of $P_{\mathrm{s}}$ in the deuterated crystal (see [2]). The optical rotatory power of the ferroelectric phase cannot be related directly to the value of $P_{\mathrm{s}}$, as suggested by Helmerbracht and Unruh [7]. The discussion of the relationship between $\mathrm{OA}$ and $P_{\mathrm{s}}$ requires an estimation of the spontaneous contribution to the total value of $\rho$ in the ferroelectric phase. Because of a small range of experimentally a vailable temperatures in the high-temperature paraelectric phase of RS and DRS, and rather large uncertainty of the $\rho$ values, a separation (analytical or graphical) of the contribution related to the paraelectric phase is difficult and charged with a large uncertainty. Nevertheless, such a separation is the only 
possibility of determination of the spontaneous optical activity along the optical axis. The $\rho_{\mathrm{s}}(T)$ was determined as illustrated in Fig. 2. The spontaneous optical rotatory power was found graphically as the difference between the broken and solid lines representing the contribution of the paraelectric phase to $\mathrm{OA}$ and the total OA in the ferroelectric phase, respectively. The estimated values of $\rho_{\mathrm{s}}$ are small. For comparison with some other optically biaxial ferroelectric crystals they are collected in the Table. It is worth noting that the character of the temperature dependence of $\rho$ in the paraelectric phase is more pronounced for the LAT [21] and NAT [22] crystals from the RS family, for which the ferroelectric transition points are better separated from their temperatures of decomposition.

\section{TABLE}

Spontaneous optical rotatory power $\rho_{\mathrm{S}}$ for some optically biaxial ferroelectric crystals ( $k \|$ optical axis, $\lambda=632.8 \mathrm{~nm},\left|T_{\mathrm{c}}-T\right|=20 \mathrm{~K}$ ).

\begin{tabular}{l|c|c}
\hline \hline Crystal & $\rho_{\mathrm{s}}\left[{ }^{\circ} \mathrm{mm}^{-1}\right]$ & Refs. \\
\hline RS & 0.06 & \\
DRS & 0.08 & \\
LAT & 0.62 & {$[21]$} \\
TGS & 0.17 & {$[14,15]$} \\
DTGS & 0.23 & {$[9,15]$} \\
TGSe & 0.49 & {$[9]$}
\end{tabular}

The temperature variations of $\rho$ reflect the temperature changes of the components of the $g_{i j}$ tensor and the change of the angle $2 \mathrm{~V}$ between the optical axes as well as the temperature changes of the light refractive index. The OA of RS and DRS crystals along the optical axis in the paraelectric phase can be described by the following relation:

$$
\rho=\frac{\pi}{\lambda n_{0}}\left(g_{11} \cos ^{2} V+g_{33} \sin ^{2} V\right)
$$

where $n_{0}$ denotes the light refractive index along the optical axis, $g_{11}$ and $g_{33}$ the components of the gyration tensor $g_{i j}, V$ - the half of the angle between the optical axes and $\lambda$ - the vacuum light wavelength.

Making use of the matrices describing the gyration tensor $g_{i j}$, linear electrogyration tensor $\gamma_{i j k l}$ and the tensor describing square electrogyration $\Pi_{i j k l}$ [23-25], and taking into account the fact that in the ferroelectric phase of RS and DRS $P_{1}=P_{\mathrm{s}}$, the gyration $G$ can be expressed as

$$
G=g_{11} l_{1}^{2}+g_{22} l_{2}^{2}+g_{33} l_{3}^{2}+2 \gamma_{14} l_{2} l_{3} P_{\mathrm{s}}+\Pi_{11} l_{1}^{2} P_{\mathrm{s}}^{2}+\Pi_{12} l_{2}^{2} P_{\mathrm{s}}^{2}+\Pi_{13} l_{3}^{2} P_{\mathrm{s}}^{2},
$$

where $l_{i}$ are the direction cosines of the wave normal of the light.

In the ferroelectric phase the indicatrix of the light refractive indices rotates about the $x$-axis by an angle $\pm \varphi_{\mathrm{s}}$ as a result of the linear electrooptical effect 
induced by the spontaneous polarization. The optical plane makes an angle $\varphi_{\mathrm{s}}$ with the plane (010), which means that all three direction cosines will be different from zero: $l_{1}=\cos V, l_{2}=\sin V \sin \left( \pm \varphi_{\mathrm{s}}\right)$, and $l_{3}=\sin V \cos \left( \pm \varphi_{\mathrm{s}}\right)$. The gyration surface and the indicatrix rotate synchronously [8]. For a small angle $\varphi_{\mathrm{s}}$ we can write

$$
\varphi_{\mathrm{s}}=a_{1} P_{\mathrm{s}}
$$

The $a_{1}$ coefficient, according to the relation given in [12, 26-28], can be written as

$$
a_{1}=\frac{\gamma_{14}}{g_{22}-g_{33}}=\frac{r_{14} n_{2}^{2} n_{3}^{2}}{n_{3}^{2}-n_{2}^{2}},
$$

where $r_{14}$ and $\gamma_{14}$ describe the linear electrooptical and linear electrogyration effects, $n_{2}$ and $n_{3}$ denote the light refractive indices along the axes $y$ and $z, g_{22}$ and $g_{33}$ are the components of the gyration tensor $g_{i j}$ (see for details papers $[26-28])$.

According to Eq. (3), the gyration $G$ in the ferroelectric phase can be written as

$$
\begin{aligned}
G= & g_{11} \cos ^{2} V+g_{33} \sin ^{2} V+P_{\mathrm{s}}^{2} g_{22} a_{1}^{2} \sin ^{2} V+2 P_{\mathrm{s}}^{2} \gamma_{14} a_{1} \sin ^{2} V \\
& +P_{\mathrm{s}}^{2} \Pi_{11} \cos ^{2} V+P_{\mathrm{s}}^{2} \Pi_{13} \sin ^{2} V+P_{\mathrm{s}}^{4} \Pi_{12} a_{1}^{2} \sin ^{2} V .
\end{aligned}
$$

Thus, taking into account Eqs. (1), (4), and (5) the spontaneous optical rotatory power $\rho_{\mathrm{s}}$ along the optical axis in RS and DRS crystals can be written as

$$
\rho_{\mathrm{s}}=\Pi_{\mathrm{ef}} P_{\mathrm{s}}^{2}+\Gamma_{\mathrm{ef}} P_{\mathrm{s}}^{4}
$$

where

$$
\Pi_{\mathrm{ef}}=\frac{\pi}{\lambda n_{0}}\left[\frac{3 g_{22}-2 g_{33}}{\left(g_{22}-g_{33}\right)^{2}} \gamma_{14}^{2} \sin ^{2} V+\Pi_{11} \cos ^{2} V+\Pi_{13} \sin ^{2} V\right]
$$

is the effective coefficient of the spontaneous electrogyration determining the magnitude of the contributions of the second order with respect to $P_{\mathrm{s}}$ and

$$
\Gamma_{\mathrm{ef}}=\frac{\pi \gamma_{14}^{2} \Pi_{12} \sin ^{2} V}{\lambda n_{0}\left(g_{22}-g_{33}\right)^{2}}
$$

is the effective coefficient of the spontaneous electrogyration determining the magnitude of the contributions of the fourth order with respect to $P_{\mathrm{s}}$. Neglecting the fourth order contributions in Eq. (6), as it was proposed by Aizu [29] in his phenomenological description of $\mathrm{OA}$, and taking into account that for the crystals showing continuous phase transition $P_{\mathrm{s}} \propto\left(T_{\mathrm{c}}-T\right)^{1 / 2}$ [30], we arrive at

$$
\rho_{\mathrm{s}} \propto\left(T_{c_{2}}-T\right) .
$$

The spontaneous optical rotatory power of RS and $\mathrm{DRS}_{\mathrm{II}}$ is a linear function of $\left(T_{\mathrm{C}_{2}}-T\right)$ within the limits of experimental uncertainty. This relation is fulfilled in the temperature ranges of $\left(T_{c_{2}}-T\right)=20 \mathrm{~K}$ for RS and $\left(T_{c_{2}}-T\right)=25 \mathrm{~K}$ for $\mathrm{DRS}_{\text {II }}$ (see Fig. 4), which means that relation (9) is held for these crystals. 


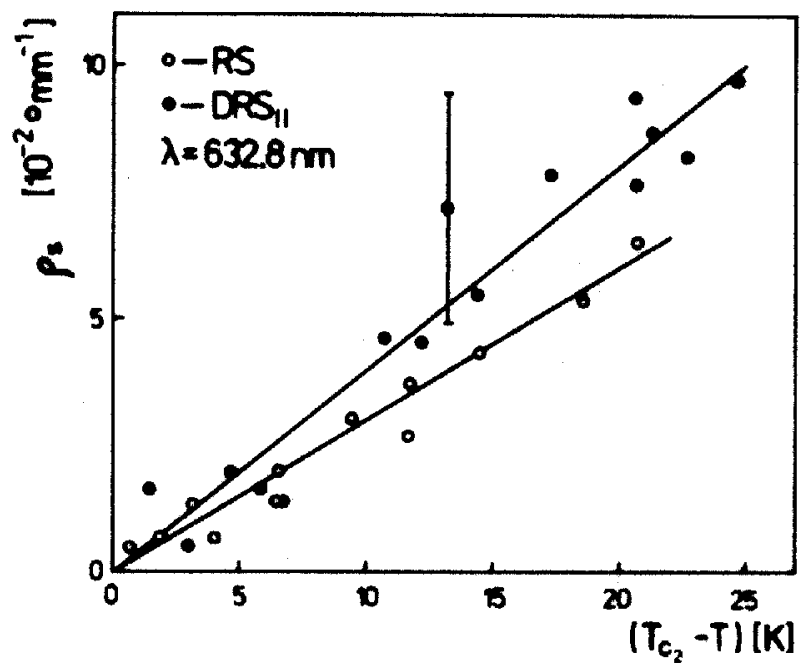

Fig. 4. The spontaneous optical rotatory power versus $\left(T_{\mathrm{C}_{2}}-T\right)$ for RS and DRS II crystals.

In view of the above, the RS and DRS crystals can be classified as belonging to the group of ferroelectrics for which the spontaneous optical rotatory power is proportional to the square of the spontaneous polarization. It has been shown that for these crystals the relation (9) is fulfilled, which means that they belong to the class of hipergyroelectrics, confirming the Aizu postulates [29].

\section{Conclusion}

(i) The changes of the OA of the RS and DRS crystals induced by an external electric field can be described by the butterfly-type hysteresis loop and the sign of $\rho$ does not change when the sense of the electric field is reversed. These findings evidence that the antipolar domains of both crystals studied are not enantiomorphic and are united by the hemitrope twin mechanism.

(ii) The temperature dependence of $\rho$ shows a continuous behaviour when the crystal passes the transition point in agreement with other experimental observations.

(iii) The spontaneous optical rotatory power $\rho_{\mathrm{S}}$ of RS and DRS is quite small and charged with high uncertainty, however, it was established that $\rho_{\mathrm{s}}$ in the first approximation depends on the square of $P_{\mathrm{s}}$.

(iv) It was found that the relation $\rho_{\mathrm{s}} \propto\left(T_{\mathrm{C}_{2}}-T\right)$ is fulfilled for both crystals studied, which means that they belong to the hipergyroelectric class as postulated by Aizu [29]. 


\section{References}

[1] J. Valasek, Phys. Rev. 17, 475 (1921).

[2] Landolt-Börnstein, Numerical Data and Functional Relationships in Science and Technology, New Series, Group III, Springer-Verlag, Berlin 1969, p. 193.

[3] P. Dufet, J. Phys. Radium 3, 757 (1904).

[4] A. Kozik, Bull. Acad. Pol. Sci., Ser. Sci. Math. Astron. Phys. A, 247 (1931).

[5] M. Koralewski, M. Mróz, Acta Phys. Pol. A 63, 507 (1983).

[6] M. Levy, Ann. Phys. (France) 5, 153 (1950).

[7] K. Hermelbracht, H.G. Unruh, Z. Angew. Physik 28, 285 (1970).

[8] O.G. Vlokh, E.A. Eazko, I.S. Zheludev, Kristallografia 20, 1056 (1975).

[9] M. Koralewski, Optical Activity and its Relation with the Ferroelectric State of Selected Ferroelectric Crystals, Ser. Phys. 73, A. Mickiewicz University Press, Poznań 2000 (in Polish).

[10] H. Iwasaki, K. Sugii, Appl. Phys. Lett. 19, 92 (1971).

[11] J. Kobayashi, K. Uchino, H. Matsuyama, K. Saito, J. Appl. Phys. 69, 409 (1991).

[12] J. Kobayashi, K. Uchino, T. Asahi, Phys. Rev. B 43, 5706 (1991).

[13] A.N. Holden, W.P. Mason, Phys. Rev. 57, 54 (1940).

[14] S. Habryło, M. Koralewski, Acta Phys. Pol. A 60, 147 (1981).

[15] M. Koralewski, S. Habryło, Ferroelectrics 46, 13 (1982).

[16] L.A. Shuvalov, K.S. Aleksandrov, I.S. Zheludev, Kristallografia 4, 130 (1959).

[17] L.A. Shuvalov, N.R. Ivanov, Kristallografia 9, 363 (1964).

[18] F. Jona, G. Shirane, Ferroelectric Crystals, Pergamon Press, Oxford 1962.

[19] W.B. Ochotnikow, A.P. Czupachin, N.Z. Ljachow, W.W. Bołchyrew, Nieorganiczeskije materialy 15, 1019 (1979) and references therein.

[20] J. Eisner, A. Miliszkiewicz, Scientific Bulletin of Opole High School of Education, No XII, Opole High School of Education Press Publ., Opole 1970, p. 29 (in Polish).

[21] M. Koralewski, M. Szafrański, Ferroelectrics 97, 233 (1989).

[22] M. Koralewski, M. Szafrański, Ferroelectrics 80, 269 (1988).

[23] J.F. Nye, Physical Properties of Crystals, PWN, Warszawa 1962 (in Polish); I.S. Zheludev, Kristallografia 9, 501 (1964).

[24] K. Aizu, Phys. Rev. 133, A1350 (1964).

[25] O.G. Vlokh, T.D. Kruszelnickaja, Kristallografia 15, 587 (1970).

[26] V.I. Indenbom, M. Czernyszewa, Kristallografia 2, 526 (1957).

[27] T. Mitsui, J. Furuchi, Phys. Rev. 90, 193 (1953).

[28] N.R. Ivanov, Izv. AN SSSR, Ser. Fiz. 48, 1162 (1984).

[29] K. Aizu, Phys. Rev. 133, A1584 (1964); ibid 134, A701 (1964).

[30] A. Devonshire, Adv. Phys. 3, 85 (1954). 